\title{
CP Violation and Extra Dimensions
}

\author{
Chao-Shang Huang ${ }^{1}$, Tianjun Li ${ }^{2}$, Liao Wei ${ }^{1}$, Qi-Shu Yan ${ }^{3}$ \\ ${ }^{1}$ ITP, Academia Sinica, P. O. Box 2735, Beijing 100080, P. R. China \\ ${ }^{2}$ Department of Physics and Astronomy, University of Pennsylvania, Philadelphia, PA \\ 19104-6396, U. S. A. \\ ${ }^{3}$ Physics Department of Tsinghua University, P. R. China
}

\begin{abstract}
It is shown that the new sources of $\mathrm{CP}$ violation can be generated in the models with more than one extra dimensions. In the supersymmetric models on the space-time $M^{4} \times T^{2} / Z_{2}$, where the radius moduli have auxiliary vacuum expectation values and the supersymmetry breaking is mediated by the Kaluza-Klein states of gauge supermultiplets, we analyze the gaugino masses and trilinear couplings for two scenarios and obtain that there exist relative $\mathrm{CP}$ violating phases among the gaugino masses and trilinear couplings.
\end{abstract}

PACS numbers: 11.30.E, 04.50, 12.60, 11.30.Q, 
It is well-known that the consistent weakly coupled (perturbative) superstring theories exist only in ten dimensions because of anomaly cancellations and the extra six dimensions must be compactified so that the universe we "see" is four-dimensional. In the weakly coupled heterotic $E_{8} \times E_{8}$ string, which was the most phenomenologically interesting candidate among the known perturbative superstring theories, the compactification energy scale is the unification scale $M_{G U T} \sim 2 \times 10^{16} \mathrm{GeV}$, which is too high to be probed. Recently, the study of the strongly coupled superstring theories and duality opens a way which leads to the possibility to have large extra dimension compactifications due to the presence of the brane. For example, in the type I/I' string theory [1] the six compact dimensions are separated into tangent and transverse to the D-branes and the string scale can be made much smaller (e.g., $1 \mathrm{TeV}$ ) than the Planck scale if the physical volume of the transverse dimensions are very large [2]. Consistent with the compactification picture of string theories, various models with large extra dimensions have been proposed and their phenomenological implications in particle physics, gravity and astrophysics have extensively been examined [3] since the pioneered works [4] were published.

On the other hand, the origin of $\mathrm{CP}$ violation has been one of main issues in high energy physics since the discovery of CP violation in the $K_{0}-\bar{K}_{0}$ system in 1964 [5]. The observation of $\operatorname{Re}\left(\epsilon^{\prime} / \epsilon\right)$ by KTeV collaboration [6] definitely confirms the earlier NA31 experiment [7]. This direct $\mathrm{CP}$ violation measurement in the kaon system can be accommodated by the CKM phase in the Standard Model within theoretical uncertainties. Recently the results on CP violation in $B_{d}-\bar{B}_{d}$ mixing have been reported by the BaBar and Belle Collaborations [8] in the ICHEP2000 Conference, which can also be explained in the Standard Model within both theoretical and experimental uncertainties. However, the CKM phase is not enough to explain the matterantimatter asymmetry in the universe and gives the contribution to the electric dipole moments (EDMs) of the neutron and electron much smaller than the experimental bounds of the EDMs of electron and neutron. One needs to have new sources of $\mathrm{CP}$ violation in addition to the $\mathrm{CP}$ violation from CKM matrix, which has been one of the motivations to search new theoretical models beyond the Standard Model, and examine their phenomenological effects.

Although vast phenomenological implications have been studied in various models with large extra dimensions, the $\mathrm{CP}$ violation' has not been examined so far. In this letter, we shall show that it is possible to have a new source of CP violation due to the presence of extra dimensions. Assuming the 6-dimensional space-time manifold is $M^{4} \times T^{2} / Z_{2}$, we calculate the gaugino masses and trilinear couplings for two scenarios in a framework where the radius moduli, which are related to the physical sizes of the extra dimensions, have auxiliary vacuum expectation values, and the supersymmetry breaking is mediated by the Kaluza-Klein states of gauge supermultiplets. We obtain that there are relative $\mathrm{CP}$ violating phases among the gauigno masses and trilinear couplings. Furthemore, it is easy to generalize our scenarios to the supersymmetric models with more than two large extra dimensions and the CP violation can be induced by similar mechanism. Therefore, we conclude that the new sources of CP violation can be generated in the supersymmetric models with more than one large extra dimensions where radius moduli have auxiliary vacuum expectation values and the supersymmetry breaking is mediated by the Kaluza-Klein states of gauge supermultiplets. Note that in 5-dimensional SUSY theories, there is no non-trivial CP violation induced due to the SUSY breaking because

\footnotetext{
${ }^{1}$ There are papers in which the $\mathrm{CP}$ violation is discussed in the models with extra dimensions [9]. However, in their models, the origin of $\mathrm{CP}$ violation is of complex vacuum expectation values of Higgs fields, which is not directly related to extra dimensions.
} 
the overall phase can be rotated away [10, 11.

For our purpose, we consider a $\mathrm{N}=1$ 6-dimensional supersymmetric (SUSY) theory compactified on $T^{2} / Z_{2}$. Upon compactification, in the 4-dimensional effective theory there are two modulus superfields, $X_{1}$ and $X_{2}$, which are related to the radii $R_{1}$ and $R_{2}$ of the torus. We assume that the observable sector is located at one of the four different fixed points of the orbifold $T^{2} / Z_{2}$ and the modulus superfields have auxiliary vacuum expectation values, $\left\langle X_{a}\right\rangle=M_{a}+\theta^{2} F_{a}$, where $\mathrm{a}=1,2$ and $M_{a} \sim R_{a}^{-1}$. We shall consider two scenarios: (A) the Standard Model gauge fields all propagate in the bulk; (B) the $\mathrm{SU}(3)$ and $\mathrm{SU}(2) \times U(1)$ propagate in different extra dimensions. In scenario $\mathrm{A}$, we obtain that there are relative $\mathrm{CP}$ violating phases between the gaugino masses and the trilinear couplings, and among the trilinear couplings. In scenario B, we also have different phases among the gaugino masses, in addition to those in scenario A.

As pointed out in Ref. [12], before Weyl-rescaling there are no (non-derivative) direct couplings of the modulus $X_{a}$ to the observable sector. The reason is that $X_{a}$ can only couple to the higher-dimensional components of the energy-momentum tensor and the wave functions of KK zero-mode fields do not depend on the extra-dimensional coordinates. The couplings of the KK (non-zero) modes of gauge supermultiplets to modulus fields will give the mass splitting in the SUSY multiplets. Thus, the KK excitations of gauge supermultiplets act as messenger fields which transmit the SUSY breaking effect to the observable sector and consequently the soft terms are generated at a quantum level in the four-dimensional effective theory. We shall use the method given in Ref. [13], i.e., from wave function renormalization, to derive the soft terms.

First, we consider the scenario A. Without loss of generality, we assume $M_{2}<M_{1}$. The messenger mass spectra can be derived from the couplings of KK excitations of gauge supermultiplets to the background superfields

$$
\int d \theta^{2} d \bar{\theta}^{2} \sum_{a=1}^{2} X_{a}^{+} e^{n_{a} V^{n_{1}, n_{2}}} X_{a}, \quad n_{1}, n_{2}=0,1,2, \ldots
$$

where $V^{n_{1}, n_{2}}$ is the KK mode with mass

$$
m_{n_{1}, n_{2}}^{2}=\sum_{i=1}^{2} \frac{n_{i}^{2}}{R_{i}^{2}}
$$

The gaugino masses are given by

$$
\tilde{M}_{i}(\mu)=-\left.\frac{1}{2} \sum_{a=1,2} \frac{\partial \ln S_{i}\left(X_{a}, \mu\right)}{\partial \ln X_{a}}\right|_{X_{a}=M_{a}} \frac{F_{a}}{M_{a}},
$$

where $i=1,2,3$, which correspond to the Standard Model gauge groups $\mathrm{U}(1), \mathrm{SU}(2)$ and $\mathrm{SU}(3)$, respectively.

Taking into account the contributions of KK excitations propagating in extra dimensions, we express the running gauge couplings as

$$
\begin{gathered}
\alpha_{i}^{-1}(\mu)=\alpha_{i}^{-1}(\Lambda)-\frac{\left(b_{i}-\tilde{b}_{i}\right)}{2 \pi} \ln \frac{\mu}{\Lambda}-\frac{\tilde{b}_{i}}{4}\left[\frac{\mu^{2}}{M_{1} M_{2}}-\frac{\Lambda^{2}}{M_{1} M_{2}}\right], \text { for } M_{1}<\mu \leq \Lambda, \\
\alpha_{i}^{-1}(\mu)=\alpha_{i}^{-1}\left(M_{1}\right)-\frac{\left(b_{i}-\tilde{b}_{i}\right)}{2 \pi} \ln \frac{\mu}{M_{1}}-\frac{\tilde{b}_{i}}{\pi}\left[\frac{\mu}{M_{2}}-\frac{M_{1}}{M_{2}}\right], \text { for } M_{2}<\mu \leq M_{1},
\end{gathered}
$$


where $\Lambda$ is the cutoff scale of the effective theory, $b_{a}$ and $\tilde{b}_{a}$ are the one-loop beta function coefficients in the MSSM and those arising from the massive gauge boson multiplets [14], respectively.

From Eqs. (3), (4) and (5), it is straightforward to derive the gaugino masses

$$
\tilde{M}_{i}(\mu)=\frac{\alpha_{i}(\mu)}{4 \pi} \tilde{b}_{i}\left[\frac{N(\Lambda)}{2}\left(\frac{F_{1}}{M_{1}}+\frac{F_{2}}{M_{2}}\right)+\left(\frac{\pi}{2}-2\right) \frac{M_{1}}{M_{2}}\left(\frac{F_{1}}{M_{1}}-\frac{F_{2}}{M_{2}}\right)-\frac{F_{2}}{M_{2}}\right],
$$

where $\mu<M_{2}$ and

$$
N(\mu) \simeq \begin{cases}\pi \mu^{2} / M_{1} M_{2}, & \text { for } M_{1}<\mu \leq \Lambda, \\ 2 \mu / M_{2}, & \text { for } M_{2}<\mu \leq M_{1},\end{cases}
$$

which is proportional to the surface of an ellipse with long axis $R_{2}$ and short axis $R_{1}$, indicating the number of the KK excitations.

Trilinear terms, $A_{Q}$, can be derived from the wave-function renormalization of chiral superfield Q, and can be expressed as

$$
A_{Q}(\mu)=\left.\sum_{a=1,2} \frac{\partial \ln Z_{Q}\left(X_{a}, X_{a}^{\dagger}, \mu\right)}{\partial \ln X_{a}}\right|_{X_{a}=M_{a}} \frac{F_{a}}{M_{a}},
$$

where $Z_{Q}$ can be solved out from the differential equations

$$
\frac{d}{d t} \ln Z_{Q}=\sum_{j=1,2,3} \frac{C_{Q}(j)}{2 \pi} \tilde{\gamma}_{Q}^{j}(\mu)
$$

where

$$
\tilde{\gamma}_{Q}^{j}(\mu)= \begin{cases}\alpha_{j}(\mu), & \text { for } \mu<M_{2}, \\ \alpha_{j}(\mu) N(\mu), & \text { for } M_{2}<\mu<\Lambda,\end{cases}
$$

where $C_{Q}(1), C_{Q}(2), C_{Q}(3)$ are the quadratic Casimirs of the $\mathrm{Q}$ representations of $\mathrm{U}(1), \mathrm{SU}(2)$, $\mathrm{SU}(3)$, respectively $\left(C_{Q}(j)=\left(j^{2}-1\right) /(2 j)\right.$ for an $\mathrm{SU}(\mathrm{j})$ fundamental.).

To simplify the discussion, we only illustrate the contribution of one gauge interaction, i.e., one term in the sum in Eq. (9). Then we have

$$
\begin{aligned}
A_{Q}^{i}(\mu)= & \frac{C_{q i}}{4 \pi}\left\{\left(\alpha_{i}\left(M_{2}\right)\left(1-\frac{\tilde{b}_{i}}{b_{i}}\right)+\frac{\tilde{b}_{i}}{b_{i}} \alpha_{i}(\mu)\right)\left[\frac{N(\Lambda)}{2}\left(\frac{F_{1}}{M_{1}}+\frac{F_{2}}{M_{2}}\right)+\left(\frac{\pi}{2}-2\right) \frac{M_{1}}{M_{2}}\left(\frac{F_{1}}{M_{1}}-\frac{F_{2}}{M_{2}}\right)-\frac{F_{2}}{M_{2}}\right]\right. \\
& \left.-\left(\frac{b_{i}}{\tilde{b}_{i}}-1\right)\left[\frac{1}{2 D^{i}}-\alpha_{i}\left(M_{2}\right)\right] \frac{F_{1}}{M_{1}}\right\},
\end{aligned}
$$

where

$$
\bar{D}^{i}=\alpha^{-1}(\Lambda)+\frac{\tilde{b}_{i}}{4} \frac{\Lambda^{2}}{M_{1} M_{2}}-\frac{b_{i}-\tilde{b}_{i}}{4 \pi} \ln \frac{M_{1} M_{2}}{\Lambda^{2}}+\frac{b_{i}-\tilde{b}_{i}}{4 \pi} .
$$

For the case $M_{1}=O\left(M_{2}\right)$, where the running between $M_{2}$ and $M_{1}$ can be neglected, the gaugino masses can be approximated to be

$$
\tilde{M}_{i}(\mu)=\frac{\alpha_{i}(\mu)}{4 \pi} \tilde{b}_{i}\left[\frac{N(\Lambda)}{2}\left(\frac{F_{1}}{M_{1}}+\frac{F_{2}}{M_{2}}\right)-\frac{\pi}{2} \frac{M_{2}}{M_{1}}\left(\frac{F_{1}}{M_{1}}-\frac{F_{2}}{M_{2}}\right)-\frac{F_{2}}{M_{2}}\right]
$$


and the trilinear term reduces to

$$
A_{Q}^{i}(\mu) \doteq \frac{C_{q i}}{b_{i}}\left[\left[1+\frac{\alpha_{i}\left(M_{2}\right)}{\alpha_{i}(\mu)}\left(\frac{b_{i}}{\tilde{b}_{i}}-1\right)\right] \tilde{M}_{i}(\mu)+\left(\frac{b_{i}}{\tilde{b}_{i}}-1\right) \frac{\alpha_{i}\left(M_{2}\right)}{8 \pi} b_{i} \frac{F_{2}}{M_{2}}\right]
$$

So, the gaugino masses and trilinear terms will have different CP violating phases when $N(\Lambda)$ is not much larger than one so that the last term of Eq. (14) could compete with the first one. This implies that the $\mathrm{CP}$ violating effects is non-trivial. In addition, we notice that among the trilinear terms, the relative $\mathrm{CP}$ violating phases can also be generated for the up-type quark, down-type quark, and lepton have different charges under the Standard Model gauge groups. Therefore, we conclude that the quantum effects can induce non-trivial CP violating phases.

Now we turn to the scenario $\mathrm{B}$, in which the $\mathrm{SU}(3)$ and $\mathrm{SU}(2) \times \mathrm{U}(1)$ gauge superfields live in different extra dimensions. The calculations are straightforward and we only give the results here. The gaugino masses are

$$
\begin{gathered}
\tilde{M}_{3}\left(M_{1}\right)=\frac{\alpha_{3}\left(M_{1}\right)}{4 \pi} \tilde{b}_{3} N_{1}(\Lambda) \frac{F_{1}}{M_{1}}, \\
\tilde{M}_{1,2}\left(M_{2}\right)=\frac{\alpha_{1,2}\left(M_{2}\right)}{4 \pi} \tilde{b}_{1,2} N_{2}(\Lambda) \frac{F_{2}}{M_{2}},
\end{gathered}
$$

where $N_{1,2}(\mu) \simeq 2\left(\mu / M_{1,2}\right)$, indicating the number of excited KK modes.

As for trilinear couplings $A_{Q}$, we obtain

$$
\begin{aligned}
& A_{D}=\sum_{q=D, Q} \alpha_{3}(\mu) \frac{C_{q 3}}{2 \pi} N_{1}(\Lambda) \frac{F_{1}}{M_{1}}+\sum_{q=D, Q} \sum_{i=1,2} \alpha_{i}(\mu) \frac{C_{q i}}{2 \pi} N_{2}(\Lambda) \frac{F_{2}}{M_{2}} \\
& A_{U}=\sum_{q=U, Q} \alpha_{3}(\mu) \frac{C_{q 3}}{2 \pi} N_{1}(\Lambda) \frac{F_{1}}{M_{1}}+\sum_{q=U, Q} \sum_{i=1,2} \alpha_{i}(\mu) \frac{C_{q i}}{2 \pi} N_{2}(\Lambda) \frac{F_{2}}{M_{2}} \\
& A_{E}=\sum_{q=E, L} \sum_{i=1,2} \alpha_{i}(\mu) \frac{C_{q i}}{2 \pi} N_{2}(\Lambda) \frac{F_{2}}{M_{2}}
\end{aligned}
$$

where $C_{U 2}=C_{D 2}=0$.

If $F_{1}$ and $F_{2}$ had different phases, we obtain that the phase and magnitude of $A_{E}$ are different from those of $A_{D}$ and $A_{U}$ from Eqs. (17-19), and the phase and magnitude of $\tilde{M}_{3}$ are different from those of $\tilde{M}_{1}$ and $\tilde{M}_{2}$ from Eqs. (15) and (16).

In summary, we have shown by two specific scenarios that the new sources of CP violation can be generated in the supersymmetric models on the space-time $M^{4} \times T^{2} / Z_{2}$. In general, provided that the number of extra dimensions is larger than one, there are new sources of $\mathrm{CP}$ violation in the supersymmetric models where the radius moduli have auxiliary vacuum expectation values and the supersymmetry breaking is mediated by the Kaluza-Klein modes of gauge supermultiplets. It should be pointed out that in the general supersymmetric theories, although in many cases the sizes of $\mathrm{CP}$ violating phases are strongly constrained by the experimental bounds on the electric dipole moments (EDMs) of the electron, neutron and ${ }^{199} \mathrm{Hg}$ atom, the possible cancellations among the different contributions to the EDMs can significantly weaken the upper bounds on the phases, therefore, the CP violating phases in the supersymmetric theories can be large [15]. 
Another possibility to have $\mathrm{CP}$ violation is to construct the model in which the charge conjugation is conserved but the parity symmetry may be broken using extra dimensions [16].

Of course, it is interesting to search other new sources of CP violation in the models with large extra dimensions.

Note added: after finishing the work, we noticed the e-preprint by Branco et al [17], in which the CP violation in quark sector in AS scenario is discussed.

\section{Acknowledgments}

The work was supported in part by the Natural Science Foundation of China and by the U.S. Department of Energy under Grant No. DOE-EY-76-02-3071.

\section{References}

[1] J. Polchinski and E. Witten, Nucl. Phys. B460 (1996) 525; for a recent reviews, see A. Sen, hep-th/9802051, I. Antoniadis and G. Ovarlez, hep-th/9906108.

[2] I. Antoniadis, hep-th/9909212.

[3] For a recent reviews, see P. Nath, hep-ph/0011177.

[4] I. Antoniadis, Phys. Lett. B246 (1990) 377; N. Arkani-Hamed, S. Dimopoulos and G. Dvali, Phys. Lett. B429 (1998) 263, ibid, B436 (1998) 257.

[5] Christensen et al., Phys. Rev. Lett. 13 (1964) 138.

[6] A. Alavi-Harati et al., Phys. Rev. Lett. 83 (1999) 22.

[7] G.D. Barr et al., NA31 collaboration, Phys. Lett. B317 (1993) 233.

[8] Plenary talks presented by D. Hitlin (BaBar Collaboration) and H. Aihara (Belle Collaboration) at ICHEP2000, Osaka, Japan, 31 July 2000 (to appear in the Proceedings).

[9] Y. Sakamura, hep-ph/9909454, 9912511.

[10] Z. Chacko and M.A. Luty, hep-ph/0008103.

[11] T. Kobayashi and K. Yoshioka, Phys. Rev. Lett. , hep-ph/0008069.

[12] M.A. Luty and R. Sundrum, Phys. Rev. D62 (2000) 035008.

[13] G. F. Giudice and R. Rattazzi, Nucl. Phys. B511, 25 (1998) hep-ph/9706540.

[14] K.R. Dienes, E. Dudas, and T. Gherghetta, Phys. Lett. B436 (1998) 55, Nucl. Phys. B537 (1999) 47. 
[15] Chao-Shang Huang and Liao Wei, Phys. Rev. D61 (2000) 116002, ibid, D62 (2000) 016008; M.Brhlik,G.J.Good, and G.L.Kane, Phys. Rev. D59,11504(1999); T.Ibrahim and P.Nath, Phys. Lett. B418 98 (1998), Phys.Rev. D57, 478(1998), (E) ibid, D58, 019901(1998), Phys. Rev. D58,111301(1998), hep-ph/9910553; T. Falk and K. Olive, Phys. Lett. B439, 71(1998); S. Pokorski, J. Rosiek, and C. Savoy, hep-ph/9906206; V. Barger, T. Falk, T. Han, J. Jiang, T. Li, T. Plehn, hep-ph/0101106.

[16] R.N. Mohapatra and A. Pérez-Lorenzana, hep-ph/9909389.

[17] G.C. Branco, A. Gouvêa and M.N. Rebelo, hep-ph/0012289. 